\title{
The Effectiveness of a Multimedia Messaging Service Reminder System in the Management of Knee Osteoarthritis: A Pilot Study
}

\author{
Gali Dar1,2*, Yaron Marx ${ }^{3}$, Emma Ioffe4, Einat Kodesh ${ }^{1}$ \\ ${ }^{1}$ Physical Therapy Department, Faculty of Social Welfare and Health Sciences, University of Haifa, Haifa, Israel \\ ${ }^{2}$ Ribstein Center for Research and Sports Medicine, Wingate Institute, Netanya, Israel \\ ${ }^{3}$ Maccabi Health Care Services, Afula and Tiberias, Israel \\ ${ }^{4}$ Maccabi Health Care Services, Grand Canyon, Haifa, Israel \\ Email: galidar@yahoo.com
}

Received 16 February 2014; revised 15 March 2014; accepted 10 April 2014

Copyright (C) 2014 by authors and Scientific Research Publishing Inc.

This work is licensed under the Creative Commons Attribution International License (CC BY). http://creativecommons.org/licenses/by/4.0/

\section{Open Access}

\section{Abstract}

Background: Patient compliance to home exercise programs is significantly linked to improved treatment outcomes. Finding ways of encouraging patient conformity to these programs is imperative. For patients with osteoarthritis $(\mathrm{OA})$ of the knee, a condition that causes pain, disability and lessens the quality of life, exercise is essential for effective control of the condition. Aim: to investigate the effectiveness of multimedia messaging services (MMS) in improving patient adherence and functional outcome to home based exercise programs for patients suffering from knee $\mathrm{OA}$. Methods: Fourteen patients diagnosed with knee $\mathrm{OA}$ and were referred to an exercise group therapy (for a total of six sessions) participated in this pilot study. The patients were randomly assigned to either the research or control group. The research group received MMS messages additional to the exercise sessions (video of exercises up to 10 seconds in length) to their mobile phone. Outcome measurement included the Western Ontario and McMaster Universities Arthritis Index (WOMAC) questionnaire, combined Focus On Therapeutic Outcomes (FOTO) questionnaire, Visual Analog Scale for Pain (VAS), Fear-Avoidance Beliefs Questionnaire (FABQ) and a general questionnaire. Results: The research group had baseline scores representing slightly higher disability, pain and fear avoidance than the control group as observed by the lower FOTO score and the higher FABQ, VAS and WOMAC scores. Analyzing the difference between initial and final scores revealed that the research group had a slightly higher perceived functional improvement. Conclusions: This study addressed the feasibility of short video messaging via mobile phones in increasing compliance to home exercise programs prescribed to patients suffering from knee $\mathrm{OA}$. This 
pilot study provides an indication for the potential of success and a larger sample study should be conducted.

Keywords

Osteoarthritis, Multimedia, Physical therapy, Rehabilitation, Exercises

\section{Introduction}

Patient compliance to home exercise programs is significantly linked to improved treatment outcomes. Finding ways of encouraging patient conformity to these programs is imperative. Indeed, in a recent Israeli demographic study, it was found that compliance with self-exercise programs was one of the strongest predictors of functional outcome [1]. Exercise is essential for effective control of osteoarthritis (OA) of the knee, a condition that causes pain, disability and poor quality of life [2]. It had been shown that physical therapy and exercise are essential for improving function, decreasing pain and reducing the need for surgical intervention among OA patients [3] [4]. However, OA sufferers were more likely to maintain a supervised rather than home exercise program [5].

Self-exercise programs clash with patient compliance as they are intrinsically time consuming and often require patience and perseverance [1]. Forgetfulness and lack of motivation are common reasons for non-adherence [6]. Short message service (SMS) texting is a constructive approach of directly addressing the problem of forgetfulness and perhaps a way of reinforcing motivation [6]. When the patient is convinced that it is worth the time and effort, good compliance with exercise programs can be achieved [1].

Health care professionals have limited contact time with their patients, therefore, finding effective and efficient means of teaching and reinforcing self-responsibility for rehabilitation should be a goal. The last decade has seen a technological revolution in communication and the universality of mobile phones. Currently, with phones that allow messaging of both text and video, an opportunity has opened up for health professionals to maintain remote contact with patients between visits. Mobile or cellular phones have become a ubiquitous appliance. In developed countries such as the United States, mobile phone use is similar across socioeconomic backgrounds [7] [8].

In recent years, SMS text messaging has been proven to be effective in all niches of health related fields. Studies have confirmed the usefulness of SMS text messaging in improving recall of rehabilitation goals [9], monitoring glucose levels in diabetic patients [10] and weight loss programs [11]. Armstrong et al. (2009) [12] demonstrated that daily text messaging was an effective way of improving adherence to daily sunscreen use. Mobile phones, internet- based motivation and action support systems have also been shown to significantly increase and maintain the level of physical activity in healthy adults [13]. Worth noting is that older populations were just as likely to favor SMS text message reminders [14].

Telemedicine is an attractive means of complimenting conventional patient-practitioner contact as it is readily accessible (and rapidly becoming more so) to most people in all sectors of the community. It is relatively inexpensive, discreet and if set up on an automated system, requires minimal time of the health practitioner [7]. The ever increasing number of applications on mobile phones (including GPS, music, internet etc.) indicates the possibilities for more advanced telemedicine which will continue to expand and provide innovative means of meeting current challenges. Davalos et al. (2009) wrote a comprehensive review of the aspects related to the economics of telemedicine. Of note, they stressed that there is a lack of economic studies to date and those addressing economic issues usually consider at the cost of running the telemedicine program without fully accounting for its benefits [15].

Telemedicine may also aid physiotherapists by providing a way of reminding patients to execute their exercises (via SMS text messaging) and possibly increase patient motivation and interest by using video messaging to demonstrate the exercises. The use of multimedia messaging services (MMS) in encouraging compliance of home exercise programs is a simple and cost effective method with potential to increase patient satisfaction and improve treatment outcomes. However, the effectiveness of SMS/MMS reminder messages to carry out physiotherapist prescribed home exercises on patient compliance and functional improvement has not been reported in the literature. 
This research aims to investigate the usefulness of MMS video messaging in improving patient adherence and functional outcome in accordance with home based exercise programs for patients suffering from OA of the knee.

\section{Materials and Methods}

\subsection{Participants}

Patients with OA of the knee were recruited from a physiotherapy group exercise class given at an out-patient clinic of Maccabi Health Services in Haifa, Israel. Patients identified as suitable for the study were randomly assigned to either the research group (who during the course of their participation in the exercise group therapy received MMS messages) or the control group (who did not receive MMS messages). Inclusion criteria included an orthopedic diagnosis of knee OA and at least a moderate competency in using mobile phone and opening MMS messages. The Helsinki Committee of Maccabi Health Services approved this study. Eligible patients read and signed an informed consent form prior to enrollment.

\subsection{Procedures}

The knee exercise group met once a week under the instruction of a physiotherapist with referred patients able to join at any stage and participate for a total of six sessions. Participants in the research group received MMS short video messages (up to 10 seconds in length) to their mobile phones every second day (excluding Saturday) with a visual reminder as to one of the home exercises prescribed at the group session (a verbal explanation, given by a physiotherapist, accompanied the visual video demonstration). A library of 12 MMS messages was created and messages were chosen arbitrarily from this library.

\subsection{Outcome Measurement}

Prior to the first group session, all participants (research and control groups) filled out the following questionnaires: Western Ontario and McMaster Universities Arthritis Index (WOMAC), combined Focus on Therapeutic Outcomes (FOTO), Visual Analog Scale for Pain (VAS), Fear-Avoidance Beliefs Questionnaire (FABQ) and a general questionnaire. At the final session, the patients filled out the same series of questionnaires. The aim of the questionnaires was to measure pain and function as well as home compliance of the exercise program and effectiveness of MMS messages in motivation.

Detailed descriptions of the questionnaires:

FOTO (Focus on Therapeutic Outcomes): FOTO is a web-based short assessment of the functional level of the impaired body part, returning a score between 0 - 100. The score represents a percentage of the functional status of the impaired body part relative to a healthy patient, i.e. a score of 30 would indicate that the patient had only $30 \%$ of the ability compared to a healthy knee. A higher score signified high function or better outcome.

FABQ (Fear-Avoidance Beliefs Questionnaire): The FABQ questionnaire was developed to assess avoidance behavior resulting from fear of pain [16]. The questionnaire consists of two subscales: FABQPA—questions relating to physical activity and FABQW-questions relating to work. A lower score signified lower fear avoidance.

VAS (Visual Analog Scale for Pain): This scale was used to assess the severity of current or recent pain with a higher score corresponding to greater pain intensity. Patients were asked to respond on a scale of 0 to 10 , the amount of pain felt, with a higher number corresponding to greater pain.

WOMAC (Western Ontario and McMaster Universities Arthritis Index): WOMAC is a standard questionnaire used to evaluate patients with OA of the knee and hip [17], assessing pain, joint stiffness, physical function, and the social and emotional function of a person with OA in determining the overall level of disability. The questionnaire consists of 24 questions for which the patient can choose responses on a scale of 0 to 4 with 0 corresponding to "not at all” and 4 corresponding to "very much". A lower score signified high function or better outcome.

Initial General Questionnaire: This short questionnaire was designed for this study and consists of four questions: 1) level of physical activity in everyday life, 2) competency in handling a mobile phone, 3) level of expectation from the group exercise sessions and 4) perceived disability. The participants were asked to answer on a scale of 0 to 4 with 0 corresponding to "not at all” and 4 corresponding to "very much". 
Final General Questionnaire: This questionnaire was created for this study and was completed at the end of the final group exercise session. Both the research and control groups were presented with five questions aimed at assessing perceived improvement in function and usefulness of the group sessions and home exercises as well as compliance with the home exercise program. The research group was presented with an additional five questions aimed at assessing the perceived usefulness of the MMS messages in providing a reminder to perform the exercises, a reminder to correctly carry out the exercises as well as compliance to opening the MMS messages and exercising after opening the messages.

\subsection{Analysis of Data}

The statistical analysis was conducted using SPSS v.16.0. and Excel software. T test and Pearson correlation coefficients test were utilized to compare research and control groups. Significant difference for all measurements was set as $\mathrm{p}<0.05$.

\section{Results}

\subsection{Participants}

Fourteen patients were recruited to the study; 9 were assigned to the control group and 5 to the research group. The research and control groups were composed of a similar mix of sexes (67\% and 57\% female, respectively) although the research group was on average, younger - 56 years old compared to 69 in the control group. The average baseline scores for the FOTO, FABQ, VAS and WOMAC questionnaires are shown in Figure 1. In general, it can be stated that the control group had baseline scores representing slightly lower disability, pain and fear avoidance than the research group, as observed by the higher FOTO score and the lower FABQ, VAS and WOMAC scores.

\subsection{Final, Post Intervention Data}

\section{Patient Compliance}

The research group had a similar perception of the usefulness of the home exercises as did the control group. The control group had a lower perceived usefulness of reminder messages. For research group specific questions, there was an enthusiastic response when asked about the usefulness of the MMS messages as reminders for performing and correctly carrying out the exercises. The patients were asked if it was beneficial to include this service in future group sessions. The average response in all cases was that the participants valued this as "very" useful. The reported opening and immediate carrying out of exercises after opening the MMS messages corresponded with the reported exercise compliance (2/3 times per week) (Table 1).

\section{Functional Outcomes}

The average final, post intervention scores for the FOTO, FABQ, VAS and WOMAC questionnaires are

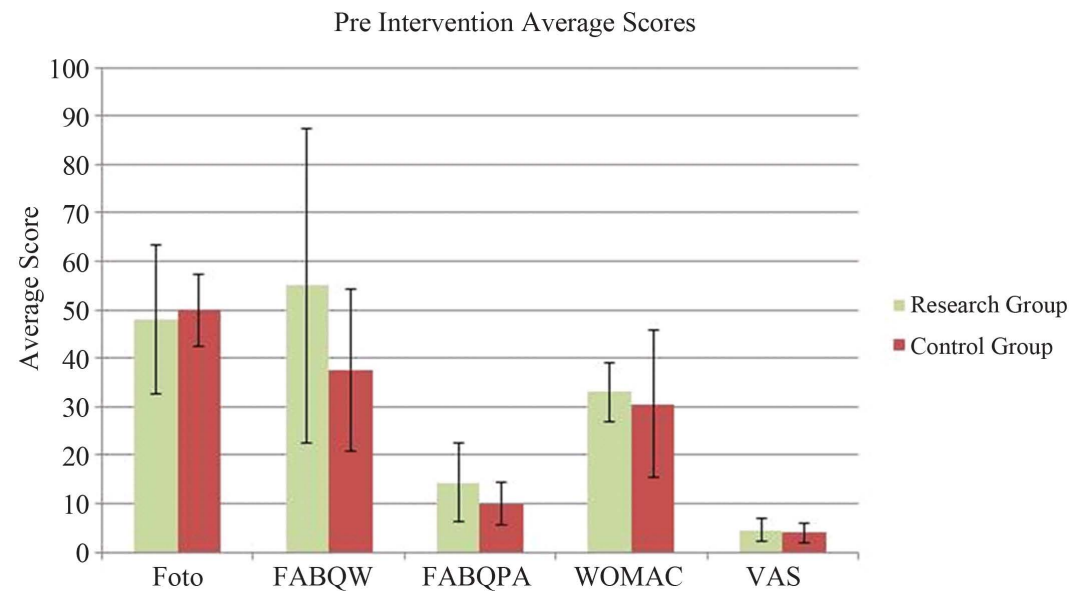

Figure 1. Average Baseline Questionnaire Scores. 
shown in Figure 2. No significant difference between groups was found as to the functional outcome indicators $(\mathrm{p}>0.05)$.

However, a more informative measure of outcome differences between the two groups was achieved by analyzing the difference between initial and final scores. The group average of this pre and post intervention score difference for each patient is shown in Figure 3. Figure 3 is displayed as an absolute value so that comparison of any outcome measure between the two groups means that a greater value corresponded to a greater functional improvement. Comparing the research and control group's average response to the final general questionnaire scores showed that the research group exhibited a slightly higher perceived functional improvement.

\section{Discussion}

This pilot study provides some indication that the use of MMS reminder messages could be effective in increasing compliance and functional outcome for patients with OA of the knee who were prescribed home exercises as part of their physiotherapy treatment. A comparison of pre and post intervention scores showed that for all out

Table 1. Response to Post Intervention General Questionnaire (empty lines questions are those which were not presented to the control group).

\begin{tabular}{|c|c|c|}
\hline \multirow{2}{*}{ Question } & \multicolumn{2}{|c|}{ Group Average Response } \\
\hline & Research & Control \\
\hline Q1) How much did your function improve? & Moderately & moderately \\
\hline Q2) How much did participation in the group sessions help you improve? & Moderately & very \\
\hline $\begin{array}{l}\text { Q3) How much do you think a personal reminder would have helped you do the exercises at } \\
\text { home? }\end{array}$ & Moderately & a little \\
\hline Q4) How much did the MMS messages help remind you to do the exercises at home? & Very & \\
\hline Q5) How much did the MMS messages help you carry out the home exercises correctly? & Very & \\
\hline Q6) How much do you feel the home exercises helped you? & Very & very \\
\hline Q7) How often did you carry out the exercises at home? & 2/3 times a week & 2/3 times a week \\
\hline Q8) How many times per week did you look at the MMS messages? & 2/3 times a week & \\
\hline $\begin{array}{l}\text { Q9) How often did you carry out the home exercises immediately after opening the MMS } \\
\text { message? }\end{array}$ & 2/3 times a week & \\
\hline Q10) Do you think it is worth including MMS messages in future groups? & Very & \\
\hline
\end{tabular}

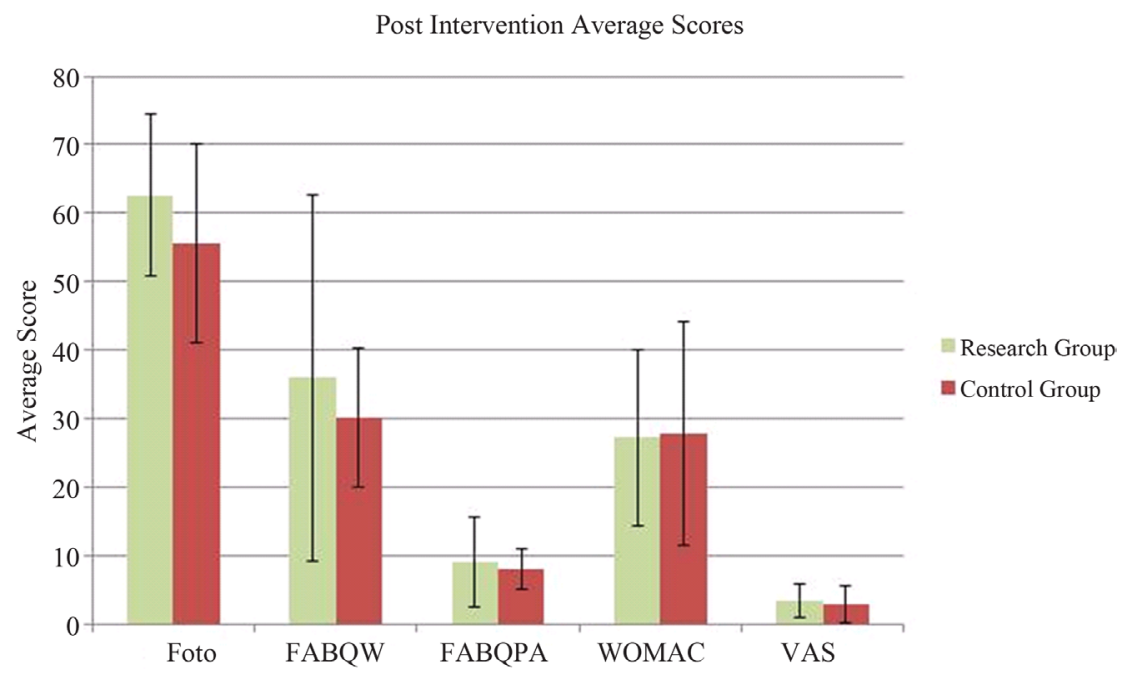

Figure 2. Average Post Intervention Questionnaire Scores. 


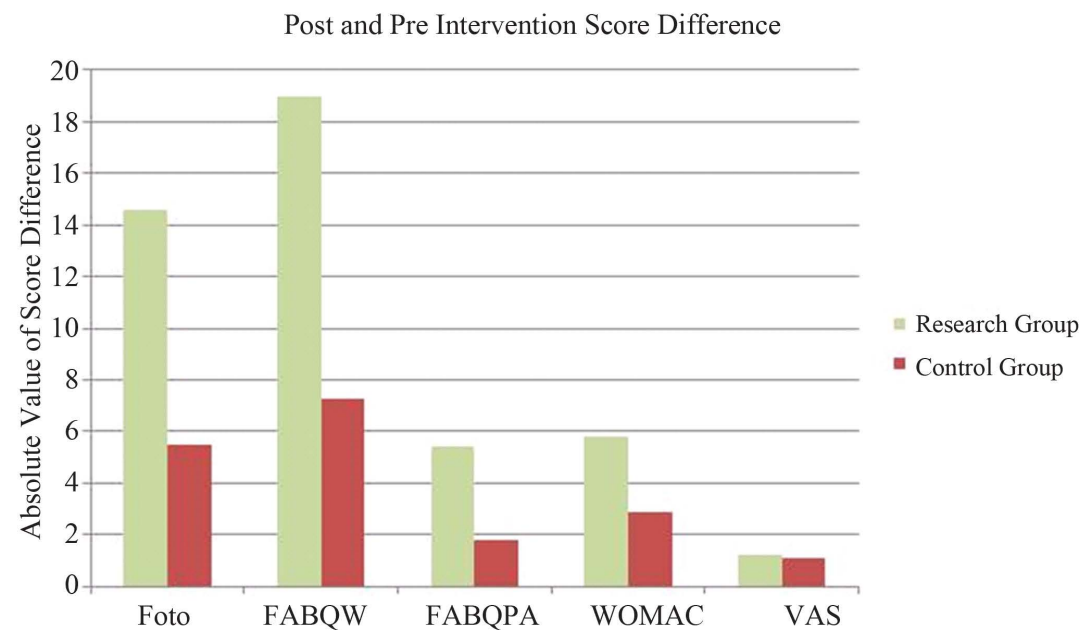

Figure 3. Absolute difference between post and pre intervention scores. Note: the actual difference in all cases was indicative of improvement.

come measures, there was more improvement in the research group than the controls (although not significantly). The outcome measures for the research group were slightly poorer than the control group on average indicatingthat the research group was more functionally impaired at the onset of the study. The research group showed greater improvement than the control group in terms of the average absolute improvement in functional outcome measures between the onset and completion of the group exercise sessions. Whether this is a result of the MMS messaging or perhaps the fact that they started with a lower functional status making improvement more attainable or noticeable, will require further investigation.

The trend for greater functional improvement for participants receiving MMS reminders together with the success of SMS and MMS reminders had been demonstrated in other medical fields. Future studies may also benefit by the use of objective measures of treatment outcome such as physiotherapist assessment. This study used only subjective measures to assess pre and post intervention, i.e. questionnaires. In order to minimize the subjectivity of this method, several specific questionnaires were used including some which are internationally recognized.

Other multimedia applications could possibly be advantageous in effectively maximizing patient treatment outcome, i.e. e-mail reminders may be used together with or in place of MMS messages. The internet could also be utilized as a forum for providing instruction videos reminding the patients how to correctly carry out the exercises and possibly a forum for online discussions regarding topics such as difficulties in performing the exercises or tracking progress. In this modern era, the use of computers has plays an important role in daily life, even within the older generation population; its potential within the realm of telemedicine should be developed.

\section{Conclusion}

This study addressed the feasibility of short video messaging via mobile phones in increasing compliance to home exercise programs prescribed to patients suffering from OA of the knee. This population can benefit from exercise and at the same time increase motivation and compliance to treatment.

\section{Study Limitations}

The major shortcoming of this study is the small number of participants which makes it difficult to gain statistically significant results. Therefore, this study should be regarded as a pilot study providing only an indication for the potential of success on a larger scale.

\section{Acknowledgements}

The authors thank Iluz limor and Amal kiwan (Maccabi Health Care Services, Grand Canyon, Haifa, Israel) for their contribution and support of this work. 


\section{References}

[1] Deutscher, D., Horn, S., Dickstein, R., Hart, D., Smout, R., Gutvirtz, M. and Ariel, I. (2009) Associations Between Treatment Processes, Patient Characteristics, and Outcomes in Outpatient Physical Therapy Practice. Archives of Physical Medicine and Rehabilitation, 90, 1349-1363. http://dx.doi.org/10.1016/j.apmr.2009.02.005

[2] Fransen, M., Crosbie, J. and Edmonds, J. (2001) Physical Therapy Is Effective for Patients with Osteoarthritis of the Knee: A Randomized Controlled Clinical Trial. Journal of Rheumatology, 28, 156.

[3] Deyle, G., Henderson, N. and Matekel, R. (2000) Effectiveness of Manual Physical Therapy and Exercise in Osteoarthritis of the Knee. A Randomized Controlled Trial. Annals of Internal Medicine, 132, 173. http://dx.doi.org/10.7326/0003-4819-132-3-200002010-00002

[4] Bennell, K., Hinman, R., Metcalf B., Buchbinder, R., McConnell, J., McColl, G., Green, S. and Crossley, K. (2005) Efficacy of Physiotherapy Management of Knee Joint Osteoarthritis: A Randomised, Double Blind, Placebo Controlled Trial. Annals of the Rheumatic Diseases, 64, 906. http://dx.doi.org/10.1136/ard.2004.026526

[5] Chamberlain, M.A., Care, G. and Harfield, B. (1982) Physiotherapy in Osteoarthrosis of the Knees. A Controlled Trial of Hospital versus Home Exercises. Disability \& Rehabilitation, 4, 101-106.

[6] Chan, D., Lonsdale, C., Ho, P., Yung, P. and Chan, K. (2009) Patient Motivation and Adherence to Postsurgery Rehabilitation Exercise Recommendations: The Influence of Physiotherapists' Autonomy-Supportive Behaviors. Archives of Physical Medicine and Rehabilitation, 90, 1977-1982. http://dx.doi.org/10.1016/j.apmr.2009.05.024

[7] Miloh, T. and Annunziato, R (2010) Adhering to Your Non-Adherent Patients: The Challenge of Non-Compliance. Acta Paediatrica, 99, 335-337. http://dx.doi.org/10.1111/j.1651-2227.2010.01702.x

[8] Krishna, S., Austin Boren, S. and Balas, A. (2009) Healthcare via Cell Phones: A Systematic Review. Telemedicine and E-Health, 15, 231-240.

[9] Culley, C. and Evans, J. (2010) SMS Text Messaging as a Means of Increasing Recall of Therapy Goals in Brain Injury Rehabilitation: A Single-Blind Within-Subjects Trial. Neuropsychological Rehabilitation, 20, 103-119. http://dx.doi.org/10.1080/09602010902906926

[10] Kim, H.S. and Jeong, H.S. (2007) A Nurse Short Message Service by Cellular Phone in Type-2 Diabetic Patients for Six Months. Journal of Clinical Nursing, 16, 1082-1087. http://dx.doi.org/10.1111/j.1365-2702.2007.01698.X

[11] Patrick, K., Raab, F., Adams, M.A., Dillon, L., Zabinski, M., Rock, C.L., Griswold, W.G. and Norman, G.J. (2009) A Text Message-Based Intervention for Weight Loss: Randomized Controlled Trial. Journal of Medical Internet Research, 11, e1. http://dx.doi.org/10.2196/jmir.1100

[12] Armstrong, A., Watson, A., Makredes, M., Frangos, J., Kimball, A. and Kvedar, J. (2009) Text-Message Reminders to Improve Sunscreen Use: A Randomized, Controlled Trial Using Electronic Monitoring. Archives of Dermatology, 145, 1230-1236. http://dx.doi.org/10.1001/archdermatol.2009.269

[13] Hurling, R., Catt, M., De Bonj, M., Fairley, B., Hurst, T., Murray, P., Richardson, A. and Sodhi, J. (2007) Using Internet and Mobile Phone Technology to Deliver an Automated Physical Activity Program: Randomized Controlled Trial. Journal of Medical Internet Research, 9, e7. http://dx.doi.org/10.2196/jmir.9.2.e7

[14] Sahm, L., MacCurtain, A., Hayden, J., Roche, C. and Richards, H. (2009) Electronic Reminders to Improve Medication Adherence-Are They Acceptable to the Patient? Pharmacy World and Science, 31, 627-629. http://dx.doi.org/10.1007/s11096-009-9327-7

[15] Davalos, M., French, M., Burdick, A. and Simmons, S (2009) Economic Evaluation of Telemedicine: Review of the Literature and Research Guidelines for Benefit-Cost Analysis. Telemedicine and E-Health, 15, 933-949.

[16] Waddell, G., Newton, M., Henderson, I., Somerville, D. and Main, C.J. (1993) A Fear-Avoidance Beliefs Questionnaire (FABQ) and the Role of Fear-Avoidance Beliefs in Chronic Low Back Pain and Disability. Pain, 52, 157-168. http://dx.doi.org/10.1016/0304-3959(93)90127-B

[17] Bellamy, N., Buchanan, W.W., Goldsmith, C.H., Campbell, J. and Stitt, L.W. (1988) Validation study of WOMAC: A Health Status Instrument for Measuring Clinically Important Patient Relevant Outcomes to Antirheumatic Drug Therapy in Patients with Osteoarthritis of the Hip or Knee. The Journal of Rheumatology, 15, 1833-18340. 